\title{
An Intelligent Group Decision Evaluation Model with Interval- Valued Intuitionistic Fuzzy Entropy Technology for Microblog User Influence
}

\author{
Lihua Zeng, ${ }^{1}$ Tonghua Yang $\mathbb{D},{ }^{2}$ Haiping Ren $\mathbb{D}^{1},{ }^{1}$ and Neal Xiong ${ }^{3}$ \\ ${ }^{1}$ Teaching Department of Basic Subjects, Jiangxi University of Science and Technology, China \\ ${ }^{2}$ School of Vocational Education and Technology, Jiangxi Agricultural University, China \\ ${ }^{3}$ Department of Mathematics and Computer Science, Northeastern State University, USA \\ Correspondence should be addressed to Tonghua Yang; yangth883@163.com
}

Received 15 October 2020; Revised 26 November 2020; Accepted 1 December 2020; Published 17 December 2020

Academic Editor: Hongju Cheng

Copyright (c) 2020 Lihua Zeng et al. This is an open access article distributed under the Creative Commons Attribution License, which permits unrestricted use, distribution, and reproduction in any medium, provided the original work is properly cited.

\begin{abstract}
Aiming at the shortcomings of some existing interval-valued intuitionistic fuzzy entropy, this paper proposes an interval-valued intuitionistic fuzzy entropy, which contains not only the interval distance between membership and nonmembership but also the average hesitancy information. On this basis, the impact evaluation of microblog users is studied. Through the multilevel decomposition of user influence, the coverage of microblog, user interaction, and user activity are constructed as the first level indicators, and nine secondary indicators are selected as the comprehensive evaluation index system of microblog influence. Considering the highly dynamic and unstructured characteristics of social network data, the idea of interval-valued intuitionistic fuzzy is introduced to transform the evaluation of social network users' influence into interval-valued intuitionistic fuzzy multiattribute group decision-making problem. Finally, the model is applied to dynamic evaluation of Sina Weibo users' influence to verify the effectiveness of the model.
\end{abstract}

\section{Introduction}

A network is composed of several nodes and links connecting these nodes, which represents many objects and their relationship. Nowadays, there are various kinds of networks in the society, such as computer network, wireless sensor network [1-5], PSTN network [6], cable TV network, Internet of things $[7,8]$, and social network.

Social network [9-12] refers to social network services, such as blog, wiki, tag, SNS, RSS, and a series of core web2.0 applications. As can be seen from Figure 1, with the wide application of web technology and mobile Internet, social networks in China have been developing rapidly. The network has really formed a society, not just a new media, new business, and new way of communication. The biggest feature of the Internet is that individuals become the main body of the Internet. Specifically, in the future, everyone, except himself in real life, will have his own representative on the Internet. It can reflect your personality, your thoughts, and all kinds of information on the Internet. At the same time, we can also communicate with you at any time. Everyone becomes a "node" of the Internet. This is different from other networks; for example, the nodes of computer networks are computers, and the nodes of wireless sensor networks are some static or mobile sensors [13-20].

In social network, some user nodes have great influence on other user nodes, which are called "opinion leader." In 1955, Katz and Lazaxsfeld [10] described high impact users, namely, "opinion leaders," as "a person can influence people in their surroundings." Through the study of voters' voting intention in the US presidential election, Katz proposed a two-level communication theory and found that individual influences differences. A small number of "opinion leaders" or "influential individuals" affect most ordinary people. Similarly, Roch also found that a small number of influential users are the key nodes to accelerate or hinder the occurrence 


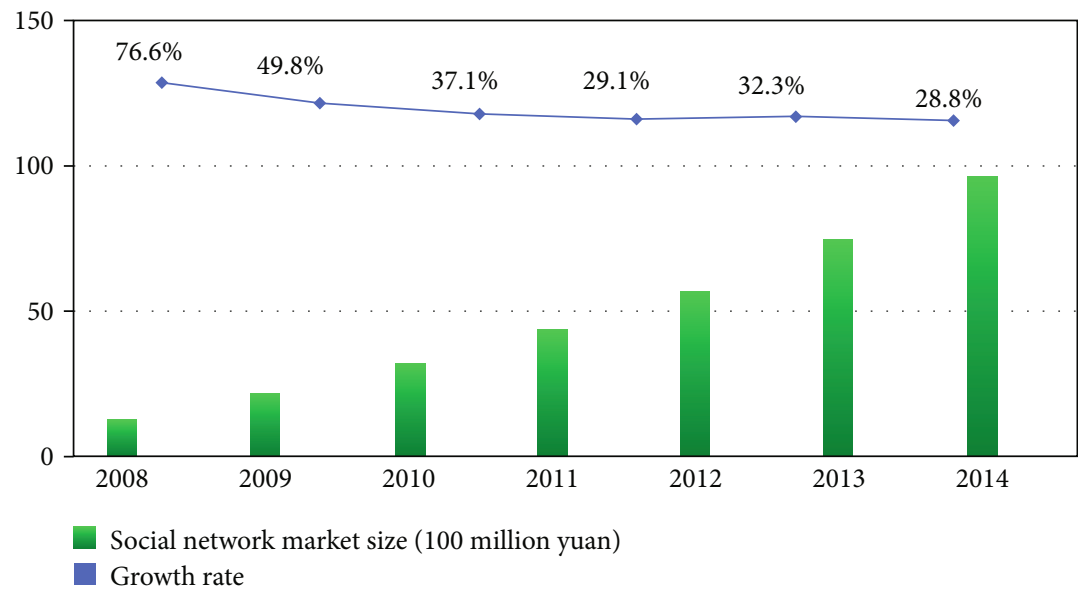

FIGURE 1: 2008-2014 China's social network market size and development trend.

of mass trading. Therefore, how to effectively evaluate the influence of users in social networks and find out "opinion leaders" play an extremely important role in information dissemination, public opinion guidance, advertising recommendation, user recommendation, and so on. In terms of public opinion monitoring, special measures should be taken for some high impact users in order to avoid public opinion flooding. For advertising, choosing the users with great influence can maximize the communication effect. As far as user recommendation is concerned, opinion leaders in the user's field of interest are often the default recommended objects. To sum up, reasonable measurement of social network users' influence is of great significance to improve the marketing effect, guide the healthy development of public opinion, and maintain the network order. This has aroused the interest of many researchers [21-26].

Atanassov and Gargov [27] extended intuitionistic fuzzy sets and proposed the concept of interval-valued intuitionistic fuzzy (IVIF) sets, which can reflect the fuzzy nature of the objective world more carefully and flexibly. IVIF entropy is a measure to describe the fuzziness of IVIF sets. It has many applications in reflecting the weight of information [28-30].

\section{The Related Work}

At present, combing the relevant literature, we find that there are various methods to measure the influence of social network users. The most commonly used methods are HITS algorithm proposed by Kleinberg [25], PageRank algorithm proposed by Page and Brin, and the improved algorithm. Hits and PageRank algorithm were proposed almost at the same time in the late 1990s and became the classic algorithm of ranking the importance of web pages in the field of search engine. Later, social network data was abstracted into directed network model by various methods, and web page ranking algorithm began to be used in social network research. Cha et al. [31] used a lot of twitter data to analyze the relationship between user behavior and influence and compared with Spearman rank correlation coefficient to get the ranking of user influence. Kwak et al. [32] used page rank algorithm to analyze the influence of users and analyzed the correlation between the number of forwarding and the number of fans and found that there was a weak correlation between them. Lee et al. [33] proposed a ranking method of influence based on time series. This method not only considers the link structure but also adds the time information of users' Publishing microblogs, which can mine some users with potential influence. Romero et al. [34] proposed a new influence measurement method IP influence after fully considering and analyzing user influence and resistance. Weng et al. [35] according to the page rank method, based on the influence of topic similarity, designed an evaluation model applied to calculate the influence of each user's microblog. Ye and $\mathrm{Wu}$ [36] proposed a method to rank the content of the user's home page as the importance ranking index by analyzing the user's behavior, the number of fans, and the number of tweets. However, there are still some defects and high complexity in web page ranking algorithm. The convergence and effectiveness of the algorithm cannot be guaranteed at the same time, and it is usually only applicable to certain types of social networks.

The third type is the user influence evaluation method based on the unified resource locator (URL) tracking. Each Internet file contains a unique URL, and its function is to locate the Internet resources. Bakshy et al. [37] calculated the influence value of each user by combining the information dissemination model and the reputation effect principle from the URL of the information release. The dynamic tracking method distinguishes the role of users in different positions on the propagation path but ignores the information difference of user behavior and communication content, which will lead to information loss.

Different from the method of user influence evaluation based on the topological structure and computer iterative operation, this paper transforms microblog user influence evaluation into the IVIF multiattribute group decisionmaking problem. The interval intuitionistic fuzzy multiattribute group decision-making method combines interval intuitionistic fuzzy theory with multiattribute group decision-making. Its feature is that the fuzzy nature of complex decision-making problems can be described more carefully by using interval numbers instead of real numbers. 
Firstly, through the multilevel decomposition of user influence, the coverage of microblog, user interaction, and user activity are constructed as the first-class indicators. Nine secondary indicators such as whether to add v-authentication, the number of microblogs, the number of active fans, the average number of daily microblogs, and the original rate of microblog are selected for the comprehensive evaluation index system of microblog influence. Secondly, time dimension is introduced to describe user data by the interval number. The advantage of IVIFN in dealing with complex, fuzzy, and uncertain data is used to transform user information data into IVIFN. Then, the IVIF entropy is improved, which includes not only the interval distance of membership and nonmembership but also the average hesitancy information. The entropy weight method is used to weight each evaluation index based on this entropy, so as to overcome the limitation of subjective weighting. Then, the criterion values are normalized and the score and precise values are calculated, so that the user influence is ranked and evaluated. Finally, the model is applied to dynamic evaluation of Sina Weibo users' influence to verify the effectiveness of the model.

\section{Preliminary Knowledge}

This section will recall some basic concepts and properties of IVIF sets.

Definition 1 [38]. Let $X_{1}$ be a given universal set. An intuitionistic fuzzy set (IFS) is an object having the form: $B=\left\{<x_{i}\right.$, $\left.\mu_{B}\left(x_{i}\right), v_{B}\left(x_{i}\right)>\mid x_{i} \in X_{1}\right\}$ where the function $\mu_{B}: X_{1} \rightarrow[0,1]$ defines the degree of membership and $v_{B}: X_{1} \rightarrow[0,1]$ defines the degree of nonmembership of the element $x_{i} \in$ $X_{1}$, respectively, and for every $x_{i} \in X_{1}$, it holds that $0 \leq \mu_{B}+$ $v_{B} \leq 1$. Furthermore, for any IFS $B$ and given $x_{i} \in X_{1}, \pi_{B}\left(x_{i}\right)$ $=1-\mu_{B}\left(x_{i}\right)-v_{B}\left(x_{i}\right)$ is called the hesitancy degree of $x_{i}$.

Definition 2 [27]. Let $X=\left\{x_{1}, x_{2}, \cdots, x_{n}\right\}$ be a universal set and $\operatorname{Int}([0,1])$ represent the set of all closed subintervals of $[0,1]$. An IVIF set is an object having the form: $\tilde{A}=\left\{<x, \tilde{\mu}_{\tilde{A}}(x)\right.$, $\left.\tilde{v}_{\tilde{A}}(x)>\mid x \in X\right\} \quad$ where the function $\tilde{\mu}_{\tilde{A}}: X \rightarrow \operatorname{Int}([0,1])$ defines the degree of membership and $\tilde{v}_{\tilde{A}}: X \rightarrow \operatorname{Int}([0,1])$ defines the degree of nonmembership of the element $x_{i} \in X$, respectively, and for every $x_{i} \in X$, it holds that $0 \leq \sup \tilde{\mu}_{\tilde{A}}(x)$ $+\sup \tilde{v}_{\tilde{A}}(x) \leq 1$. For convenience, IVIF sets can also be written as

$$
\tilde{A}=\left\{<x,\left[\mu_{\tilde{A}}^{L}(x), \mu_{\tilde{A}}^{U}(x)\right],\left[v_{\tilde{A}}^{L}(x), v_{\tilde{A}}^{U}(x)\right]>\mid x \in X\right\},
$$

where $\mu_{\tilde{A}}^{U}(x)+v_{\tilde{A}}^{U}(x) \leq 1, \mu_{\tilde{A}}^{L}(x) \geq 0$ and $v_{\tilde{A}}^{L}(x) \geq 0$.

Let $\tilde{\pi}_{\tilde{A}}(x)=1-\tilde{\mu}_{\tilde{A}}(x)-\tilde{v}_{\tilde{A}}(x)=\left[1-\mu_{\tilde{A}}^{U}(x)-v_{\tilde{A}}^{U}(x), 1-\right.$ $\left.\mu_{\tilde{A}}^{L}(x)-v_{\tilde{A}}^{L}(x)\right]$; then, it is called hesitancy of $x_{i}$. All IVIF sets on $X$ are denoted as $\operatorname{IVIFS}(X)$.

Generally, the ordered pair $\left(\left[\mu_{\tilde{A}}^{L}(x), \mu_{\tilde{A}}^{U}(x)\right],\left[\nu_{\tilde{A}}^{L}(x), v_{\tilde{A}}^{U}(x)\right]\right)$ composed of membership interval $\left[\mu_{\tilde{A}}^{L}(x), \mu_{\tilde{A}}^{U}(x)\right]$ and nonmembership interval $\left[v_{\tilde{A}}^{L}(x), v_{\tilde{A}}^{U}(x)\right]$ of element $x$ in $X$ is called IVIF number (IVIFN). For convenience, the general form of IVIFN is denoted as $([a, b],[c, d])$, where $[a, b] \subset$ $[0,1],[c, d] \subset[0,1], b+d \leq 1$.

Definition 3 [27]. Let $\tilde{A}$ and $\tilde{B}$ be two IVIF sets; then

(1) $\tilde{A} \subseteq \tilde{B}$ if and only if

$\mu_{\tilde{A}}^{L}(x) \leq \mu_{\tilde{B}}^{L} \quad(x), \mu_{\tilde{A}}^{U}(x) \leq \mu_{\tilde{B}}^{U}(x), v_{\tilde{A}}^{L}(x) \geq v_{\tilde{B}}^{L}(x), v_{\tilde{A}}^{U}(x) \geq$ $\nu_{\tilde{B}}^{U}(x)$, for all $x_{i} \in X$

(2) $\tilde{A}=\tilde{B}$ if and only if $\tilde{A} \subseteq \tilde{B}$ and $\tilde{B} \subseteq \tilde{A}$

(3) The complementary set of $\tilde{A}$ denoted by $\tilde{A}^{C}$ is

$$
\tilde{A}^{C}=\left\{<x,\left[\nu_{\tilde{A}}^{L}(x), v_{\tilde{A}}^{U}(x)\right],\left[\mu_{\tilde{A}}^{L}(x), \mu_{\tilde{A}}^{U}(x)\right]>\mid x \in X\right\}
$$

Definition 4 [27]. Let $\tilde{a}=([a, b],[c, d]), \tilde{a}_{1}=\left(\left[a_{1}, b_{1}\right],\left[c_{1}, d_{1}\right]\right)$, and $\tilde{a}_{2}=\left(\left[a_{2}, b_{2}\right],\left[c_{2}, d_{2}\right]\right)$ be three IVIFNs; then

$$
\begin{aligned}
& \text { (1) } \tilde{a}_{1}+\tilde{a}_{2}=\left(\left[a_{1}+a_{2}-a_{1} a_{2}, b_{1}+b_{2}-b_{1} b_{2}\right],\left[c_{1} c_{2}, d_{1} d_{2}\right]\right. \\
& \text { ) } \\
& \text { (2) } \lambda \tilde{a}=\left(\left[1-(1-a)^{\lambda}, 1-(1-b)^{\lambda}\right],\left[c^{\lambda}, d^{\lambda}\right]\right), \lambda>0 \\
& \text { (3) } \tilde{a}^{\lambda}=\left(\left[a^{\lambda}, b^{\lambda}\right],\left[1-(1-c)^{\lambda}, 1-(1-d)^{\lambda}\right]\right), \lambda>0
\end{aligned}
$$

Definition 5. Let $\tilde{a}=([a, b],[c, d])$ be an IVIFN. The score function and accuracy function of $\tilde{a}$ are

$$
\begin{aligned}
& \Delta(\tilde{a})=\frac{1}{4}(a-c+b-d)\left(1+\frac{1}{a+b-a c+b d}\right), \\
& H(\tilde{a})=\frac{1}{2}(a+b+c+d),
\end{aligned}
$$

respectively, which are given by Gao et al. [28].

Based on the score and accuracy functions, a comparison law for IVIFN is introduced by Gao et al. [28] as follows:

Let $\tilde{a}_{1}$ and $\tilde{a}_{2}$ be two IVIFNs, $\Delta\left(\tilde{a}_{1}\right)$ and $\Delta\left(\tilde{a}_{2}\right)$ be the scores of $\tilde{a}_{1}$ and $\tilde{a}_{2}$, respectively, $H\left(\tilde{a}_{1}\right)$ and $H\left(\tilde{a}_{2}\right)$ be the accuracy degrees of $\tilde{a}_{1}$ and $\tilde{a}_{2}$, respectively.

(1) If $\Delta\left(\tilde{a}_{1}\right)<\Delta\left(\tilde{a}_{2}\right)$, then $\tilde{a}_{1}<\tilde{a}_{2}$.

(2) If $\Delta\left(\tilde{a}_{1}\right)=\Delta\left(\tilde{a}_{2}\right)$, then

$$
\left\{\begin{array}{l}
H\left(\tilde{a}_{1}\right)=H\left(\tilde{a}_{2}\right) \Rightarrow \tilde{a}_{1}=\tilde{a}_{2}, \\
H\left(\tilde{a}_{1}\right)<H\left(\tilde{a}_{2}\right) \Rightarrow \tilde{a}_{1}<\tilde{a}_{2}
\end{array}\right.
$$

Definition 6 [29]. Let $\tilde{a}_{j}=\left(\left[a_{j}, b_{j}\right],\left[c_{j}, d_{j}\right]\right)(j=1,2, \cdots, n)$ be a collection of IVIFNs and $\omega=\left(\omega_{1}, \omega_{2}, \cdots, \omega_{n}\right)^{T}$ be the weight vector of $\tilde{a}_{j}(j=1,2, \cdots, n)$, where $\omega_{j}$ indicates the importance degree of $\tilde{a}_{j}$, satisfying $\omega_{j} \geq 0(j=1,2, \cdots, n)$ and $\sum_{j=1}^{n}$ $\omega_{j}=1$, and let IIFWA ${ }_{\omega}: F_{I}^{n} \rightarrow F_{I}$, if 


$$
\begin{aligned}
\operatorname{IIFWA}_{\omega}\left(\tilde{a}_{1}, \tilde{a}_{2}, \cdots, \tilde{a}_{n}\right)=( & {\left[1-\prod_{j=1}^{n}\left(1-a_{j}\right)^{\omega_{j}}, 1-\prod_{j=1}^{n}\left(1-b_{j}\right)^{\omega_{j}}\right], } \\
& {\left.\left[\prod_{j=1}^{n} c_{j}^{\omega_{j}}, \prod_{j=1}^{n} d_{j}^{\omega_{j}}\right]\right), }
\end{aligned}
$$

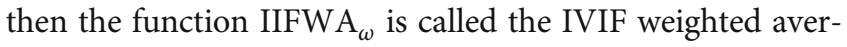
age operator.

Definition 7 [38]. Let $\tilde{A}=\left\{<x,\left[\mu_{\tilde{A}}^{L}(x), \mu_{\tilde{A}}^{U}(x)\right],\left[v_{\tilde{A}}^{L}(x), v_{\tilde{A}}^{U}(x)\right]\right.$ $>\mid x \in X\}$ be an IVIF set. The hesitation of $\tilde{A}$ is $\pi_{\tilde{A}}(x)=\left[\pi_{\tilde{A}}^{L}\right.$ $\left.(x), \pi_{\tilde{A}}^{U}(x)\right]$, where $\pi_{\tilde{A}}^{L}(x)=1-\mu_{\tilde{A}}^{U}(x)-v_{\tilde{A}}^{U}(x)$ and $\pi_{\tilde{A}}^{U}(x)=$ $1-\mu_{\tilde{A}}^{L}(x)-v_{\tilde{A}}^{L}(x)$. We define the average hesitation $\Psi_{\tilde{A}}(x)$ of $\tilde{A}$ as follows:

$\Psi_{\tilde{A}}(x)=\frac{\pi_{\tilde{A}}^{L}(x)+\pi_{\tilde{A}}^{U}(x)}{2}=\frac{2-\mu_{\tilde{A}}^{L}(x)-\mu_{\tilde{A}}^{U}(x)-v_{\tilde{A}}^{L}(x)-v_{\tilde{A}}^{U}(x)}{2}$.

Here, $\Psi_{\tilde{A}}(x)$ expresses the lack of information.

We define a distance between membership and nonmembership of element $x$ in $X$, that is,

$$
\Phi_{\tilde{A}}(x)=\frac{1}{2}\left|\mu_{\tilde{A}}^{L}(x)-v_{\tilde{A}}^{L}(x)\right|+\frac{1}{2}\left|\mu_{\tilde{A}}^{U}(x)-v_{\tilde{A}}^{U}(x)\right| .
$$

$\Phi_{\tilde{A}}(x)$ describes the balance between membership and nonmembership of element $x$.

Definition 8 [38]. Let $\tilde{A}$ be an IVIF set defined on $X$. IVIF entropy is defined as a real valued function $E(\tilde{A})=f\left(\Psi_{\tilde{A}}\right.$, $\left.\Phi_{\tilde{A}}\right): \operatorname{IVIFS}(X) \rightarrow[0,1]$, which satisfies the following axiomatic conditions:

(i) $E(\tilde{A})=0$ if and only if $\tilde{A}$ is a crisp set, i.e. $\tilde{\mu}_{\tilde{A}}(x)=$ $[1,1], \tilde{v}_{\tilde{A}}(x)=[0,0]$ or $\tilde{\mu}_{\tilde{A}}(x)=[0,0], \tilde{v}_{\tilde{A}}(x)=[1,1]$, $\forall x \in X$

(ii) $E(\tilde{A})=1$ if and only if $\left[\mu_{\tilde{A}}^{L}(x), \mu_{\tilde{A}}^{U}(x)\right]=\left[\nu_{\tilde{A}}^{L}(x), v_{\tilde{A}}^{U}\right.$ $(x)], \forall x \in X$

(iii) $E(\tilde{A})=E\left(\tilde{A}^{c}\right)$

(iv) $E(\tilde{A})=f\left(\Psi_{\tilde{A}}, \Phi_{\tilde{A}}\right)$ is a real valued continuous function and is increasing with respect to parameter $\Psi_{\tilde{A}}$ and decreasing with respect to parameter $\Phi_{\tilde{A}}$

\section{A New IVIF Entropy}

Before proposing a new IVIF entropy, we first analyze the limitations of some previous IVIF entropy.

(1) One is that the uncertainty of IVIF is not considered

For example, the entropy formula of Vlachos and Sergiadis [39] is as follows:
$E_{\mathrm{VS}}(\tilde{A})=1-\sqrt{\frac{1}{2} \sum_{i=1}^{n}\left(\left(\left|\mu_{\tilde{A}}^{L}\left(x_{i}\right)-v_{\tilde{A}}^{L}\left(x_{i}\right)\right|\right)^{2}+\left(\left|\mu_{\tilde{A}}^{U}\left(x_{i}\right)-v_{\tilde{A}}^{U}\left(x_{i}\right)\right|\right)^{2}\right)}$.

(2) The other is that although the influence of hesitancy on entropy is considered. There are still some cases that cannot be distinguished well

Example 8. Let $\tilde{A}_{1}=([0.15,0.25],[0.1,0.2])$ and $\tilde{B}_{1}=([0.35$, $0.45],[0.3,0.4])$ be two IVIFNs.

Obviously, the degree of fuzziness of $\tilde{A}_{1}$ is greater than $\tilde{B}_{1}$. We use formula (7) to calculate as follows:

$$
\begin{aligned}
E_{\mathrm{VS}}\left(\tilde{A}_{1}\right) & =1-\sqrt{\frac{1}{2}\left(|0.15-0.1|^{2}+|0.25-0.2|^{2}\right)}=0.95, E_{\mathrm{VS}}\left(\tilde{B}_{1}\right) \\
& =1-\sqrt{\frac{1}{2}\left(|0.35-0.3|^{2}+|0.45-0.4|^{2}\right)}=0.95 .
\end{aligned}
$$

Then, $E_{\mathrm{VS}}\left(\tilde{A}_{1}\right)=E_{\mathrm{VS}}\left(\tilde{B}_{1}\right)$ which is obviously inconsistent with the facts.

The IVIF entropy measure defined by Zhang et al. [40] is as follows:

$E_{Z}(\tilde{A})=\frac{1}{n} \sum_{i=1}^{n} \frac{\min \left\{\mu_{\tilde{A}}^{L}\left(x_{i}\right), v_{\tilde{A}}^{L}\left(x_{i}\right)\right\}+\min \left\{\mu_{\tilde{A}}^{U}\left(x_{i}\right), v_{\tilde{A}}^{U}\left(x_{i}\right)\right\}}{\left.\mu_{\tilde{A}}^{L}\left(x_{i}\right), v_{\tilde{A}}^{L}\left(x_{i}\right)\right\}+\max \left\{\mu_{\tilde{A}}^{U}\left(x_{i}\right), v_{\tilde{A}}^{U}\left(x_{i}\right)\right\}}$.

Example 9. Let $\tilde{A}_{2}=([0.3,0.5],[0.1,0.3])$ and $\tilde{B}_{2}=([0.1,0.3]$, $[0,0.2])$ be two IVIFNs. Obviously, the degree of fuzziness of $\tilde{B}_{2}$ is greater than $\tilde{A}_{2}$. We use formula (11) to calculate as follows:

$$
\begin{aligned}
& E_{Z}\left(\tilde{A}_{2}\right)=\frac{0.1+0.3}{0.3+0.5}=0.5, \\
& E_{Z}\left(\tilde{B}_{2}\right)=\frac{0+0.2}{0.1+0.3}=0.5 .
\end{aligned}
$$
facts.

$E_{Z}\left(\tilde{A}_{2}\right)=E_{Z}\left(\tilde{B}_{2}\right)$ which is obviously inconsistent with the

Example 10. Let $\tilde{A}_{3}=([0.1,0.3],[0,0.5])$ and $\tilde{B}_{3}=([0.2,0.4]$ , $[0.1,0.6])$ be two IVIFNs. Obviously, the degree of fuzziness of $\tilde{A}_{3}$ is greater than $\tilde{B}_{3}$. The IVIF entropy measure defined by Ren and Tan [41] is as follows:

$E_{R}(\tilde{A})=\frac{1}{n} \sum_{i=1}^{n} \frac{4-\left[\left|\mu_{\tilde{A}}^{L}\left(x_{i}\right)-v_{\tilde{A}}^{L}\left(x_{i}\right)\right|+\left|\mu_{\tilde{A}}^{U}\left(x_{i}\right)-v_{\tilde{A}}^{U}\left(x_{i}\right)\right|\right]^{2}+\left|\pi_{\tilde{A}}^{L}\left(x_{i}\right)-\pi_{\tilde{A}}^{U}\left(x_{i}\right)\right|^{2}}{8}$.

The result of calculation is $E_{R}\left(\tilde{A}_{3}\right)=E_{R}\left(\tilde{B}_{3}\right)=0.4275$, which is obviously inconsistent with the facts. 
In order to make up for the deficiency of the existing IVIF entropy, we will construct a new IVIF entropy.
Let $\tilde{A}=\left\{<x_{i},\left[\mu_{\tilde{A}}^{L}\left(x_{i}\right), \mu_{\tilde{A}}^{U}\left(x_{i}\right)\right],\left[v_{\tilde{A}}^{L}\left(x_{i}\right), v_{\tilde{A}}^{U}\left(x_{i}\right)\right]>\mid x_{i} \in X\right\}$ be an IVIF set. We define

$$
E_{Q}(\tilde{A})=\frac{1}{n} \sum_{i=1}^{n} \cos \frac{\left(\left|\mu_{\tilde{A}}^{L}\left(x_{i}\right)-v_{\tilde{A}}^{L}\left(x_{i}\right)\right|+\left|\mu_{\tilde{A}}^{U}\left(x_{i}\right)-v_{\tilde{A}}^{U}\left(x_{i}\right)\right|\right)\left(2-\pi_{\tilde{A}}^{L}\left(x_{i}\right)-\pi_{\tilde{A}}^{U}\left(x_{i}\right)\right)}{8} \pi,
$$

$E_{Q}(\tilde{A})$ can be rewritten as

$$
E_{Q}(\tilde{A})=\frac{1}{n} \sum_{i=1}^{n} \cos \frac{\left((1 / 2)\left|\mu_{\tilde{A}}^{L}\left(x_{i}\right)-v_{\tilde{A}}^{L}\left(x_{i}\right)\right|+(1 / 2)\left|\mu_{\tilde{A}}^{U}\left(x_{i}\right)-v_{\tilde{A}}^{U}\left(x_{i}\right)\right|\right)\left(1-\left(\left(\pi_{\tilde{A}}^{L}\left(x_{i}\right)+\pi_{\tilde{A}}^{U}\left(x_{i}\right)\right) / 2\right)\right)}{2} \pi=\frac{1}{n} \sum_{i=1}^{n} \cos \frac{\Phi_{\tilde{A}}\left(x_{i}\right)\left(1-\Psi_{\tilde{A}}\left(x_{i}\right)\right)}{2} \pi
$$

Obviously, $E_{\mathrm{Q}}(\tilde{A})$ contains not only the interval distance $\Phi_{\tilde{A}}(x)$ of membership and nonmembership but also the information of average hesitation degree $\Psi_{\tilde{A}}(x)$.

Theorem 11. The measure $E_{Q}(\tilde{A})$ defined by equation (14) is an IVIF entropy.

Proof. To prove the measure $E_{Q}(\tilde{A})$ given by equation (14) is an IVIF entropy, we only need to prove it satisfies the properties in Definition 8. Obviously, for every $x_{i}$, we have:

$$
0 \leq \mu_{\tilde{A}}^{L}\left(x_{i}\right), \mu_{\tilde{A}}^{U}\left(x_{i}\right), v_{\tilde{A}}^{L}\left(x_{i}\right), v_{\tilde{A}}^{U}\left(x_{i}\right), \pi_{\tilde{A}}^{L}\left(x_{i}\right), \pi_{\tilde{A}}^{U}\left(x_{i}\right) \leq 1,
$$

then,

$0 \leq \frac{\left(\left|\mu_{\tilde{A}}^{L}\left(x_{i}\right)-v_{\tilde{A}}^{L}\left(x_{i}\right)\right|+\left|\mu_{\tilde{A}}^{U}\left(x_{i}\right)-v_{\tilde{A}}^{U}\left(x_{i}\right)\right|\right)\left(2-\pi_{\tilde{A}}^{L}\left(x_{i}\right)-\pi_{\tilde{A}}^{U}\left(x_{i}\right)\right)}{8} \pi \leq \frac{\pi}{2}$,

thus, we have $0 \leq E_{Q}(\tilde{A}) \leq 1$.

(i) Let $\tilde{A}$ be a crisp set; i.e., for all $x_{i} \in X$, we have $\left[\mu_{\tilde{A}}^{L}\right.$ $\left.(x), \mu_{\tilde{A}}^{U}(x)\right]=[1,1],\left[v_{\tilde{A}}^{L}(x), v_{\tilde{A}}^{U}(x)\right]=[0,0]$ or $\left[\mu_{\tilde{A}}^{L}(x)\right.$, $\left.\mu_{\tilde{A}}^{U}(x)\right]=[0,0],\left[v_{\tilde{A}}^{L}(x), v_{\tilde{A}}^{U}(x)\right]=[1,1]$.

It is obviously that $E_{Q}(\tilde{A})=0$.

If $E_{Q}(\tilde{A})=0$, that is,

$$
E_{Q}(\tilde{A})=\frac{1}{n} \sum_{i=1}^{n} \cos \frac{\left(\left|\mu_{\tilde{A}}^{L}\left(x_{i}\right)-v_{\tilde{A}}^{L}\left(x_{i}\right)\right|+\left|\mu_{\tilde{A}}^{U}\left(x_{i}\right)-v_{\tilde{A}}^{U}\left(x_{i}\right)\right|\right)\left(2-\pi_{\tilde{A}}^{L}\left(x_{i}\right)-\pi_{\tilde{A}}^{U}\left(x_{i}\right)\right)}{8} \pi=0,
$$

then, for all $x_{i} \in X$, we have

$\cos \frac{\left(\left|\mu_{\tilde{A}}^{L}\left(x_{i}\right)-v_{\tilde{A}}^{L}\left(x_{i}\right)\right|+\left|\mu_{\tilde{A}}^{U}\left(x_{i}\right)-v_{\tilde{A}}^{U}\left(x_{i}\right)\right|\right)\left(2-\pi_{\tilde{A}}^{L}\left(x_{i}\right)-\pi_{\tilde{A}}^{U}\left(x_{i}\right)\right)}{8} \pi=0$.

Thus,

$\left(\left|\mu_{\tilde{A}}^{L}\left(x_{i}\right)-v_{\tilde{A}}^{L}\left(x_{i}\right)\right|+\left|\mu_{\tilde{A}}^{U}\left(x_{i}\right)-v_{\tilde{A}}^{U}\left(x_{i}\right)\right|\right)\left(2-\pi_{\tilde{A}}^{L}\left(x_{i}\right)-\pi_{\tilde{A}}^{U}\left(x_{i}\right)\right)=4$,

then, we have $\left[\mu_{\tilde{A}}^{L}(x), \mu_{\tilde{A}}^{U}(x)\right]=[1,1],\left[\nu_{\tilde{A}}^{L}(x), v_{\tilde{A}}^{U}(x)\right]=[0,0]$ or $\left[\mu_{\tilde{A}}^{L}(x), \mu_{\tilde{A}}^{U}(x)\right]=[0,0],\left[v_{\tilde{A}}^{L}(x), v_{\tilde{A}}^{U}(x)\right]=[1,1]$.

Therefore, $\tilde{A}$ is a crisp set (ii) Let $\left[\mu_{\tilde{A}}^{L}\left(x_{i}\right), \mu_{\tilde{A}}^{U}\left(x_{i}\right)\right]=\left[v_{\tilde{A}}^{L}\left(x_{i}\right), v_{\tilde{A}}^{U}\left(x_{i}\right)\right], \forall x_{i} \in X$, obviously, we have $E_{Q}(\tilde{A})=1$

Now, we assume that $E_{Q}(\tilde{A})=1$; then, for all $x_{i} \in X$, we have

$$
\cos \frac{\left(\left|\mu_{\tilde{A}}^{L}\left(x_{i}\right)-v_{\tilde{A}}^{L}\left(x_{i}\right)\right|+\left|\mu_{\tilde{A}}^{U}\left(x_{i}\right)-\nu_{\tilde{A}}^{U}\left(x_{i}\right)\right|\right)\left(2-\pi_{\tilde{A}}^{L}\left(x_{i}\right)-\pi_{\tilde{A}}^{U}\left(x_{i}\right)\right)}{8} \pi=1,
$$

then, $\left(\left|\mu_{\tilde{A}}^{L}\left(x_{i}\right)-v_{\tilde{A}}^{L}\left(x_{i}\right)\right|+\left|\mu_{\tilde{A}}^{U}\left(x_{i}\right)-v_{\tilde{A}}^{U}\left(x_{i}\right)\right|\right)\left(2-\pi_{\tilde{A}}^{L}\left(x_{i}\right)-\pi_{\tilde{A}}^{U}\right.$ $\left.\left(x_{i}\right)\right)=0$; we can obtain the conclusion $\left[\mu_{\tilde{A}}^{L}\left(x_{i}\right), \mu_{\tilde{A}}^{U}\left(x_{i}\right)\right]=$ $\left[v_{\tilde{A}}^{L}\left(x_{i}\right), v_{\tilde{A}}^{U}\left(x_{i}\right)\right]$ or $\left[\pi_{\tilde{A}}^{L}\left(x_{i}\right), \pi_{\tilde{A}}^{U}\left(x_{i}\right)\right]=[1,1]$ for all $x_{i} \in X$. If $\left[\pi_{\tilde{A}}^{L}\left(x_{i}\right), \pi_{\tilde{A}}^{U}\left(x_{i}\right)\right]=[1,1]$, we can also get $\left[\mu_{\tilde{A}}^{L}\left(x_{i}\right), \mu_{\tilde{A}}^{U}\left(x_{i}\right)\right]$ $=\left[v_{\tilde{A}}^{L}\left(x_{i}\right), v_{\tilde{A}}^{U}\left(x_{i}\right)\right]$ for all $x_{i} \in X$ 
TABLE 1: Calculation results of IVIF entropy for some IVIFNs.

\begin{tabular}{lccr}
\hline Case & IVIFNs & IVIF entropy & Results \\
\hline \multirow{2}{*}{ Case 1 } & $\tilde{A}_{1}=([0.15,0.25],[0.1,0.2])$ & $E_{Q}\left(\tilde{A}_{1}\right)=0.9996$ & $\tilde{A}_{1}>\tilde{B}_{1}$ \\
& $\tilde{B}_{1}=([0.35,0.45],[0.3,0.4])$ & $E_{Q}\left(\tilde{B}_{1}\right)=0.9983$ & $\tilde{B}_{2}>\tilde{A}_{2}$ \\
\hline \multirow{2}{*}{ Case 2 } & $\tilde{A}_{2}=([0.3,0.5],[0.1,0.3])$, & $E_{Q}\left(\tilde{A}_{2}\right)=0.9851$ & $\tilde{A}_{3}>\tilde{B}_{3}$ \\
& $\tilde{B}_{2}=([0.1,0.3],[0,0.2])$ & $E_{Q}\left(\tilde{B}_{2}\right)=0.9989$ & \\
\hline
\end{tabular}

(iii) By equation (14), we have

$$
E_{Q}\left(\tilde{A}^{C}\right)=\frac{1}{n} \sum_{i=1}^{n} \cos \frac{\left(\left|v_{\tilde{A}}^{L}\left(x_{i}\right)-\mu_{\tilde{A}}^{L}\left(x_{i}\right)\right|+\left|v_{\tilde{A}}^{U}\left(x_{i}\right)-\mu_{\tilde{A}}^{U}\left(x_{i}\right)\right|\right)\left(2-\pi_{\tilde{A}}^{L}\left(x_{i}\right)-\pi_{\tilde{A}}^{U}\left(x_{i}\right)\right)}{8} \pi=E_{Q}(\tilde{A})
$$

(iv) Let

$$
f(x, y)=\cos \frac{y(1-x) \pi}{2}
$$

where $x, y \in[0,1]$. We need to prove the function $f(x, y)$ is increasing with $x$ and decreasing with $y$.

We take the partial derivatives of $f(x, y)$ with respect to $x$ and $y$, respectively:

$$
\begin{aligned}
& \frac{\partial f}{\partial x}=\frac{y \pi}{2} \sin \frac{y(1-x) \pi}{2}>0 \\
& \frac{\partial f}{\partial y}=-\frac{(1-x) \pi}{2} \sin \frac{y(1-x) \pi}{2}<0 .
\end{aligned}
$$

Thus, $f(x, y)$ is increasing with $x$ and decreasing with $y$. In conclusion, $E_{Q}(\tilde{A})$ is an IVIF entropy.

Remark 12. Let $\tilde{A} \succ \tilde{B}$ represent IVIFN $\tilde{A}$ which is more fuzzy than IVIFN $\tilde{B}$. Applying $E_{Q}(\tilde{A})$ to calculate the IVIF entropy of Examples 8 to 10, the results are shown in the following Table 1.

Therefore, it can be seen that the entropy $E_{Q}(\tilde{A})$ proposed in this paper is more reasonable than formulas (9), (11), and (13).

\section{Microblog User Influence Group Decision Evaluation Model}

At present, research on IVIF sets focuses on the basic theory, and there is little research on its application in multiattribute group decision-making $[27,39]$. The entropy of IVIF set is solved after the criterion type is normalized. Then, the weight is determined according to the entropy weight method. Finally, the obtained criterion value is normalized to realize the ranking and evaluation of schemes. The characteristic of this method is that interval numbers are used to describe decision information instead of real numbers.

Based on the idea of IVIF multiattribute group decisionmaking, the dynamic evaluation model of microblog user influence combines the user influence evaluation with fuzzy thought. This paper decomposes the user influence index in multilevel, establishes a comprehensive and reasonable index system, describes the user data with interval number, and introduces the time dimension to investigate the dynamic changes of data, and generates IVIF group decision matrix. After normalization according to the type of criteria, the entropy proposed in Section 2 is used to determine the criterion weight. Finally, the criterion value is normalized and the score value and accurate value are obtained. The essence of microblog user influence group decision-making evaluation model is to transform the user influence evaluation into IVIF multiattribute group decision-making problem. The entropy weight method is used to determine the weight of the criteria, and the time dimension is introduced to evaluate the user influence.

Now, we give the steps of our new evaluation model.

Step 1. Establish the decision matrix.

According to the method of multi-attribute group decision-making, its general form is as follows:

For a multiattribute group decision-making problem, let $X=\left\{x_{1}, x_{2}, \cdots, x_{n}\right\}$ be the scheme set, $C=\left\{c_{1}, c_{2}, \cdots, c_{m}\right\}$ the criterion set, and $D=\left\{d_{1}, d_{2}, \cdots, d_{l}\right\}$ the decision-maker set. If the decision maker $d_{k}$ expresses the value of scheme $x_{i}$ under criterion $c_{j}$ by interval number, the decision matrix formed is marked as 


$$
\begin{aligned}
& X_{i}=\left(\left[a_{k j}^{i}, b_{k j}^{i}\right]\right)_{l \times m}
\end{aligned}
$$

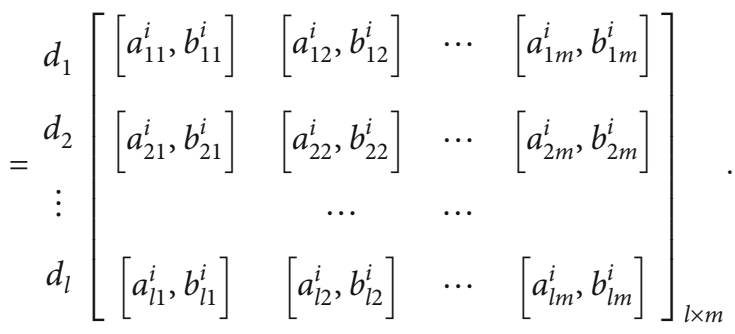

Step 2. Normalize the decision matrix.

In the uncertain network environment, social events contain different decision-making indicators. Because of their different meanings, there are dimensional differences. In order to eliminate the influence of different dimensions on decision-making, we first normalize the dimensionless data. With reference to Hu et al. [42], for different types of criteria, they are standardized according to the following formula, where $L=\{1,2, \cdots, l\}, J=\{1,2, \cdots, m\}$, and $I=\{1,2, \cdots, n\}$.

For benefit based criteria, let

$$
\left\{\begin{array}{c}
x_{k j}^{i}=\frac{a_{k j}^{i}-\min _{i}\left\{a_{k j}^{i}\right\}}{\max _{i}\left\{b_{k j}^{i}\right\}-\min _{i}\left\{a_{k j}^{i}\right\}}, \\
y_{k j}^{i}=\frac{b_{k j}^{i}-\min _{i}\left\{a_{k j}^{i}\right\}}{\max _{i}\left\{b_{k j}^{i}\right\}-\min _{i}\left\{a_{k j}^{i}\right\}}, \\
k \in L, j \in J, i \in I .
\end{array}\right.
$$

For cost-based criteria, let

$$
\left\{\begin{array}{c}
x_{k j}^{i}=\frac{\max _{i}\left\{b_{k j}^{i}\right\}-b_{k j}^{i}}{\max _{i}\left\{b_{k j}^{i}\right\}-\min _{i}\left\{a_{k j}^{i}\right\}}, \\
y_{k j}^{i}=\frac{\max _{i}\left\{b_{k j}^{i}\right\}-a_{k j}^{i}}{\max _{i}\left\{b_{k j}^{i}\right\}-\min _{i}\left\{a_{k j}^{i}\right\}} \\
k \in L, j \in J, i \in I .
\end{array}\right.
$$

The normalized decision matrix is $Y_{i}=\left(\left[x_{k j}^{i}, y_{k j}^{i}\right]\right)_{l \times m}$.

Step 3. The interval number matrix is transformed into the IVIFN matrix.

In this paper, the method proposed by Yue [43] is applied to transform interval valued to IVIFNs. For the decision matrix $Y_{i}=\left(\left[x_{k j}^{i}, y_{k j}^{i}\right]\right)_{l \times m}$, the higher the value of $\left[x_{k j}^{i}, y_{k j}^{i}\right]$, the greater the satisfaction of decision makers. Let

$$
\theta_{i j}^{L}=\min _{k}\left\{y_{k j}^{i}\right\}, \theta_{i j}^{U}=\max _{k}\left\{y_{k j}^{i}\right\}
$$

$$
\eta_{i j}^{L}=\min _{k}\left\{x_{k j}^{i}\right\}, \eta_{i j}^{U}=\max _{k}\left\{x_{k j}^{i}\right\}
$$

where $\left[\theta_{i j}^{L}, \theta_{i j}^{U}\right]$ and $\left[\eta_{i j}^{L}, \eta_{i j}^{U}\right]$ denote the satisfaction interval and dissatisfaction interval of the criterion, respectively. Let these two intervals form an ordered pair and mark them as $\left(\left[\theta_{i j}^{L}, \theta_{i j}^{U}\right],\left[\eta_{i j}^{L}, \eta_{i j}^{U}\right]\right)$. The smaller the value of $\eta_{i j}^{L}$ and $\eta_{i j}^{U}$ is, the more dissatisfied the decision maker is. If

$$
\rho_{i j}^{L}=1-\eta_{i j}^{U}, \rho_{i j}^{U}=1-\eta_{i j}^{L}
$$

then, $r_{i j}=\left(\left[\mu_{i j}^{L}, \mu_{i j}^{U}\right],\left[v_{i j}^{L}, v_{i j}^{U}\right]\right)$ is called IVIFN of criterion $c_{j}$ under scheme $x_{i}$, where

$$
\begin{aligned}
& \mu_{i j}^{L}=\frac{\theta_{i j}^{L}}{\theta_{i j}^{L}+\theta_{i j}^{U}+\rho_{i j}^{L}+\rho_{i j}^{U}}, \mu_{i j}^{U}=\frac{\theta_{i j}^{U}}{\theta_{i j}^{L}+\theta_{i j}^{U}+\rho_{i j}^{L}+\rho_{i j}^{U}}, \\
& v_{i j}^{L}=\frac{\rho_{i j}^{L}}{\theta_{i j}^{L}+\theta_{i j}^{U}+\rho_{i j}^{L}+\rho_{i j}^{U}}, v_{i j}^{U}=\frac{\rho_{i j}^{U}}{\theta_{i j}^{L}+\theta_{i j}^{U}+\rho_{i j}^{L}+\rho_{i j}^{U}} .
\end{aligned}
$$

The transformed decision matrix is recorded as $R=$ $\left(r_{i j}\right)_{n \times m}$.

Step 4. Determine the weight of criteria.

IVIF entropy is used to describe the fuzzy degree of IVIF sets. The larger the IVIF entropy of the evaluation criterion is, the greater the fuzziness degree of the information provided by the criterion is, and a smaller weight should be given. On the contrary, it gives a larger weight. The weight of each criterion can be determined by the above principle. Using the entropy formula proposed in Section 2 of this paper, if the value of a single scheme $x_{i}$ under criterion $c_{j}$ is $r_{i j}$, then, its fuzzy entropy is as follows:

$$
E\left(r_{j}\right)=\frac{1}{n} \sum_{i=1}^{n} \cos \frac{\left(\left|\mu_{i j}^{L}-v_{i j}^{L}\right|+\left|\mu_{i j}^{U}-v_{i j}^{U}\right|\right)\left(2-\pi_{i j}^{L}-\pi_{i j}^{U}\right)}{8} \pi .
$$

Then, the weight of each criterion is calculated by equation (34).

$$
\omega_{j}=\frac{1-E\left(r_{j}\right)}{\sum_{j=1}^{m}\left(1-E\left(r_{j}\right)\right)} .
$$

Step 5. Solve the comprehensive criterion value.

By using formula (6), the comprehensive criterion value $z_{i}$ of each scheme is obtained.

Step 6. Sort the results and choose the best.

According to formulas (3) and (4), the score value and accuracy value of the comprehensive criteria value are solved; that is, the influence score and accuracy value of candidate users are obtained, and the user influence is sorted according to Definition 5. 


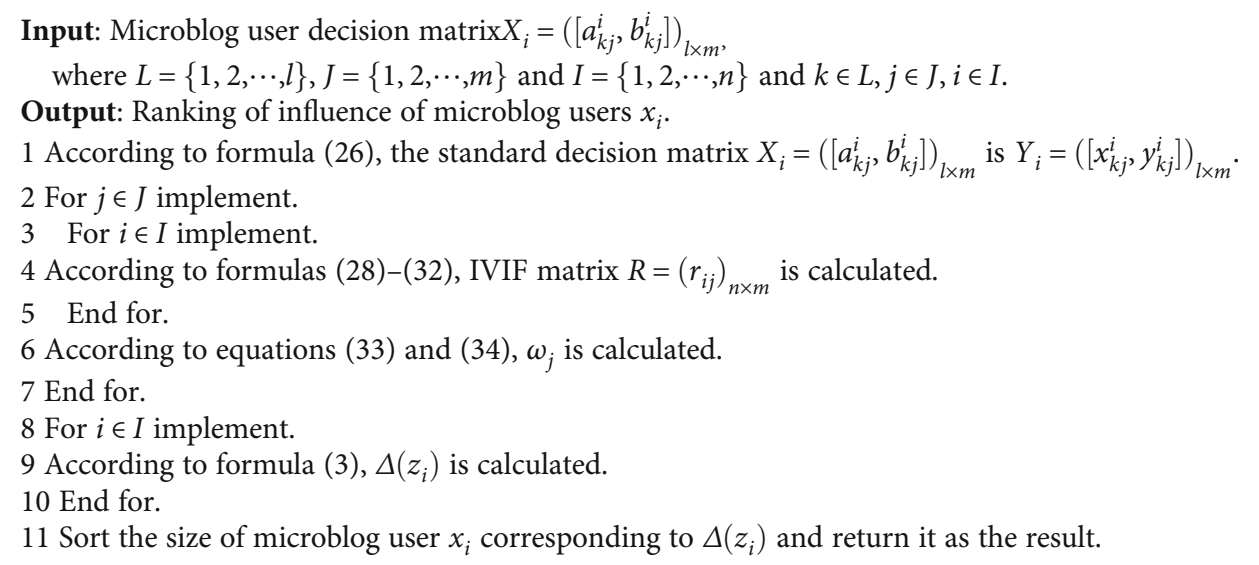

Algorithm 1: IVIFMI algorithm.

According to the above steps, we get the following algorithm, which we call interval-valued intuitionistic fuzzy microblog influence algorithm IVIFMI algorithm (see Algorithm 1).

\section{Performance Analysis}

Microblog is a broadcast social network platform based on attention mechanism to share short and real-time information. It is one of the most active social networks in China. This paper selects Sina Weibo as the research object. According to the data in the 41st statistical report on China's Internet Development released by CNNIC [9], as of December 2017, the number of microblog users in China had reached 376 million, which promoted the continuous growth of user utilization rate to $40.9 \%$, an increase of $3.8 \%$ compared with December 2016 (as shown in Figure 2).

In addition to publishing their own original microblog information, each user can also forward, comment, and like the microblog information of other users at will. The behaviors of forwarding, commenting, and liking among different users contribute to the formation of microblog information dissemination networks (as shown in Figure 3). In addition, the microblog platform also has the characteristics of low user threshold, short microblog content, and strong readability, which make the dissemination speed of microblog information faster and has a wider range of influence.

This paper selects Sina Weibo as the research object.

6.1. Data Acquisition. The datum comes from Weibo Fengyun, which is collected by crawler tools. Weibo Fengyun is an official authorized data service provider of Sina Weibo. In this paper, six Sina Weibo users were randomly sampled to track their information and collect data from July 17, 2020 , to July 25,2020 . This information can reflect the user's activities on Sina Weibo from registration to July 25, 2020.

6.2. Data Description and Index Measurement. The traditional method of measuring user influence only by the number of fans of microblog users has lost its credibility. The selection of influence index of microblog users should be comprehensively considered according to the basic functions of microblog. Through the analysis of user behavior characteristics, it is found that the influence of microblog users can be constructed from three aspects: microblog coverage, user interaction, and user activity [44]. The coverage of microblog includes three indicators: the number of active fans, the number of blog posts, and user authentication. User interaction includes three indicators: the number of comments, the number of forwarding, and the number of likes. User activity includes daily average number of microblogs, original microblog rate, and active days, as shown in Figure 4.

The meaning and measurement method of indicators are as follows:

(1) The coverage of microblog $\left(o_{1}\right)$ : this indicator includes the number of active fans $\left(o_{11}\right)$, the number of microblogs $\left(o_{12}\right)$, and user authentication $\left(o_{13}\right)$. Among them, the number of active fans and the number of microblogs are very intuitive influencing factors. The number of fans determines the scope of the user's influence, and the number of blogs determines the depth of the user's influence. For fans of the same size, the more microblogs users have, the more times each fan is affected by the user's microblog information, that is, the deeper the influence of the user is. The influence of user authentication on users is a potential factor. If users pass the authentication of Sina Weibo, the credibility of microblog is very high, which makes the possibility of the microblog being commented and forwarded increased, so the influence of users is increased.

The number of active fans refers to the number of fans who have eliminated zombie powder. It is represented by numerical data. In this paper, users with less than 20 followers and less than 10 microblogs are identified as Zombie fans.

The number of user microblogs refers to the total number of microblogs published by users' microblog accounts during data collection. It is expressed by numerical value. 


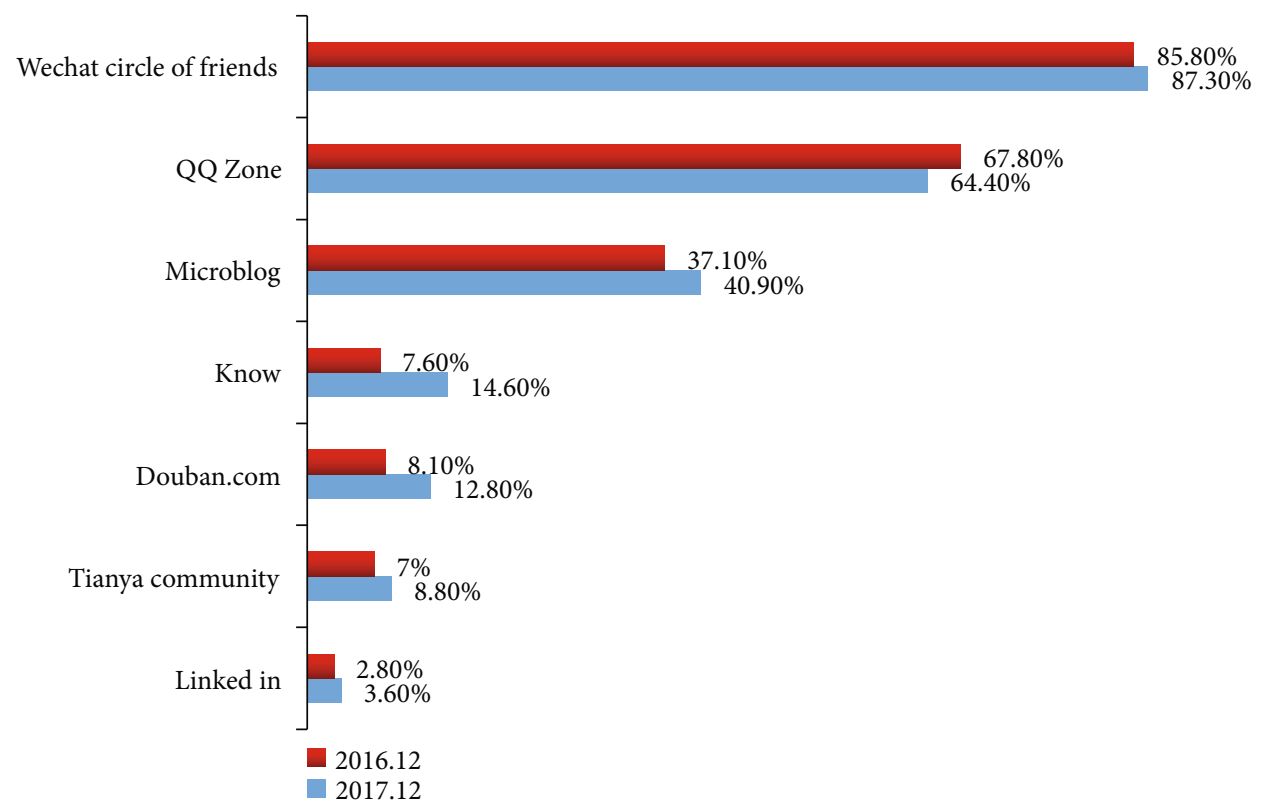

FIgURE 2: Typical social software usage.

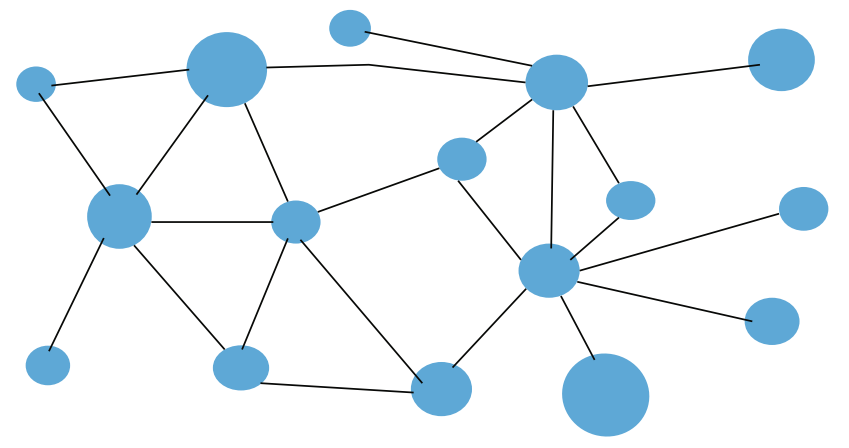

FIGURE 3: Examples of microblog information dissemination network.

Authenticated users refer to users who have been authenticated by microblog, usually marked by "V." Whether the user is authenticated is indicated by a value of 0 or 1 ( 1 indicates that the user has completed the authentication, and 0 indicates that the user has not completed the authentication).

(2) User interaction $\left(o_{2}\right)$ : this indicator includes the number of comments $\left(o_{21}\right)$, the number of forwarding $\left(o_{22}\right)$, and the number of likes $\left(o_{23}\right)$. In the microblog platform, the microblog users post and forward affect their fans, and the behavior of fans such as comments, forwarding, and likes in turn promotes the dissemination of microblog information. The more times a microblog message is forwarded, the more widely it is spread. The more comments are made, the more attention is paid to the information. The more likes a user gets, the higher the prestige of the user in his fan community.

This paper selects the average number of comments, average number of forwarding, and average number of likes of each microblog. Referring to Chen et al. [24], after data preprocessing, the number of comments is represented by an interval number $\left[a^{L}, a^{U}\right]$, the number of forwarding is represented by interval number $\left[b^{L}, b^{U}\right]$, and the number of compliments is represented by interval number $\left[c^{L}, c^{U}\right]$ (see formulas (35)-(37)).

$$
\begin{aligned}
& a^{L}=\sum_{i=1}^{n_{1}} \frac{r_{i}}{n_{1}}, a^{U}=\sum_{i=1}^{n_{2}} \frac{r_{i}}{n_{2}}, \\
& b^{L}=\sum_{i=1}^{m_{1}} \frac{s_{i}}{m_{1}}, b^{U}=\sum_{i=1}^{m_{2}} \frac{s_{i}}{m_{2}}, \\
& c^{L}=\sum_{i=1}^{k_{1}} \frac{t_{i}}{k_{1}}, c^{U}=\sum_{i=1}^{k_{2}} \frac{t_{i}}{k_{2}} .
\end{aligned}
$$

(3) User activity $\left(o_{3}\right)$ : this indicator includes daily average number of microblogs $\left(o_{31}\right)$, microblog original rate $\left(o_{32}\right)$, and active days $\left(o_{33}\right)$. The average number of daily microblogs refers to the average number of microblogs per day since users opened microblogs, which can reflect the average activity of users. Microblog can be divided into forwarding microblog and original microblog. The content of the original microblog is created by users themselves. The active days show the frequency of users participating in microblog. Referring to Chen et al.'s study [24], after data preprocessing, the daily average number of microblogs is represented by interval number $\left[x^{L}\right.$, $\left.x^{U}\right]$, and the microblog original rate is expressed by interval number $\left[y^{L}, y^{U}\right]$. The data of active days is from the basic information of microblog users and 


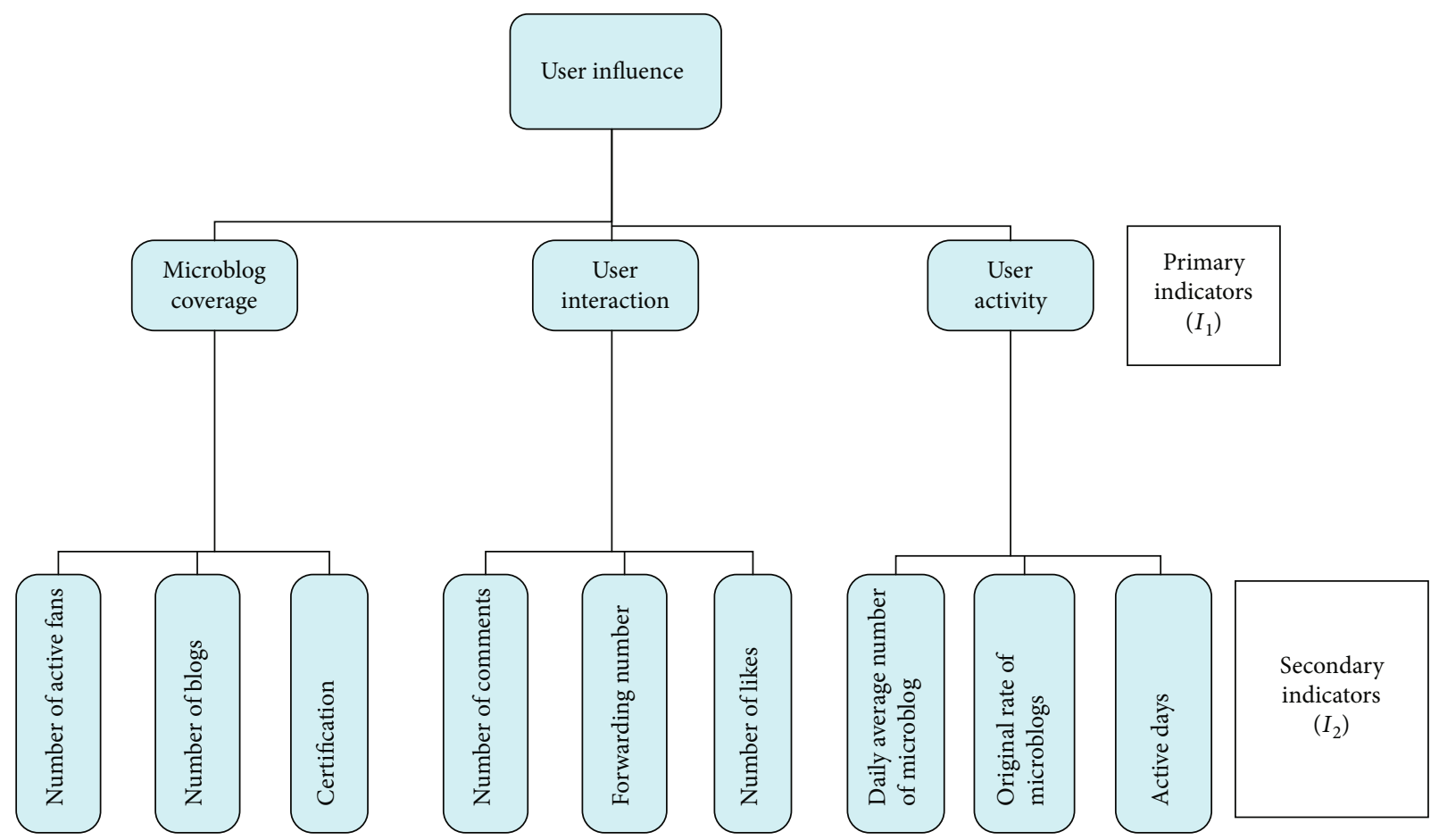

FIgURE 4: The evaluation index system of microblog user influence.

expressed by a numerical value (see formulas (38) and (39)).

$$
\begin{aligned}
& x^{L}=\sum_{i=1}^{l_{1}} \frac{x_{i}}{l_{1}}, x^{U}=\sum_{i=1}^{l_{2}} \frac{x_{i}}{l_{2}}, \\
& y^{L}=\frac{\sum_{i=1}^{j_{1}} y_{i} / j_{1}}{\bar{x}}, y^{U}=\frac{\sum_{i=1}^{j_{2}} y_{i} / j_{2}}{\bar{x}} .
\end{aligned}
$$

The meaning of the symbols in equations (35)-(39) is shown in Table 2.

Using the above data description and index measurement method, the data of user 1 is obtained, as shown in Table 3.

In the same way, the data of the other five users can be obtained. Due to the length of the article, the data of the other five users is not shown here. Data of six users constitute the decision matrix.

6.3. Calculation Results. All the indexes selected in this paper are benefit indicators, and the decision matrix obtained in the previous section is normalized by using formula (26). According to formulas (33) and (34), the weight $\left(\omega_{1}, \omega_{2}, \cdots\right.$ ,$\left.\omega_{m}\right)$ of the criterion is obtained as follows:

$$
\begin{aligned}
\omega_{1} & =0.1474, \omega_{2}=0.1296, \omega_{3}=0.0818, \omega_{4} \\
& =0.1141, \omega_{5}=0.1440, \omega_{6}=0.1244, \omega_{7} \\
& =0.0921, \omega_{8}=0.0402, \omega_{9}=0.1264 .
\end{aligned}
$$

Using formula (6), the comprehensive criterion values of each scheme are obtained as follows:

$$
\begin{aligned}
z_{1} & =([0.296,0.304],[0.204,0.274]), z_{2} \\
& =([0.356,0.465],[0.248,0.413]), z_{3} \\
& =([0.325,0.486],[0.236,0.425]), z_{4} \\
& =([0.412,0.536],[0.222,0.364]), z_{5} \\
& =([0.555,0.730],[0.167,0.270]), z_{6} \\
& =([0.456,0.680],[0.272,0.406]) .
\end{aligned}
$$

According to formula (3), the comprehensive criterion score value is as follows:

$$
\begin{aligned}
\Delta\left(z_{1}\right)= & 0.080, \Delta\left(z_{2}\right)=0.083, \Delta\left(z_{3}\right)=0.077, \Delta\left(z_{4}\right) \\
= & 0.177, \Delta\left(z_{5}\right)=0.365, \Delta\left(z_{6}\right)=0.203 .
\end{aligned}
$$

Thus, the sorting result is $x_{5}>x_{6}>x_{4}>x_{2}>x_{1}>x_{3}$. User 5 is the most influential user.

It should be noted that the quantitative model of user influence proposed in this paper is also applicable to other online social network platforms such as WeChat and QQ.

\section{Conclusion and Future Work}

In this paper, a new IVIF entropy is proposed, which contains not only the interval distance of membership and nonmembership but also the average hesitancy. On this basis, the influence of microblog users is studied. Through the multilevel decomposition of user influence, the coverage, user interaction, and user activity of microblog are constructed as the first level indicators. Nine secondary indicators such as whether to add $\mathrm{V}$ authentication, the number of microblogs, the number of active fans, the average number of 
TABLE 2: Symbols and their explanations.

\begin{tabular}{|c|c|}
\hline Symbol & Symbolic meaning \\
\hline$n$ & The total number of microblogs published by users from the time of opening microblogs to data collection \\
\hline$r_{i}(i=1,2, \cdots, n)$ & The number of comments on the $i$-th microblog \\
\hline $\bar{r}$ & The average number of comments per microblog \\
\hline$n_{1}$ & The number of microblogs of $r_{i}<\bar{r}$ \\
\hline$n_{2}$ & The number of microblogs of $r_{i}>\bar{r}$ \\
\hline$s_{i}(i=1,2, \cdots, n)$ & The forwarding number of the $i$-th microblog \\
\hline $\bar{s}$ & Average forwarding number of users per microblog \\
\hline$m_{1}$ & The number of microblogs of $s_{i}<\bar{s}$ \\
\hline$m_{2}$ & The number of microblogs of $s_{i}>\bar{s}$ \\
\hline$t_{i}(i=1,2, \cdots, n)$ & The number of likes received by users on the $i$-th microblog \\
\hline $\bar{t}$ & Average number of likes per microblog \\
\hline$k_{1}$ & The number of microblogs of $t_{i}<\bar{t}$ \\
\hline$k_{2}$ & The number of microblogs of $t_{i}>\bar{t}$ \\
\hline$l$ & The usage time of microblog, which refers to the total days from the user's account opening to data collection \\
\hline$x_{i}(i=1,2, \cdots, l)$ & The number of microblogs published by the user on the $i$-day \\
\hline $\bar{x}$ & The average number of microblogs published by the user per day \\
\hline$l_{1}$ & The total days of $x_{i}<\bar{x}$ \\
\hline$l_{2}$ & The total days of $x_{i}>\bar{x}$ \\
\hline$y_{i}(i=1,2, \cdots, l)$ & The number of original microblogs published by users on the $i$-th day \\
\hline $\bar{y}$ & Average number of original microblogs published by users per day \\
\hline$j_{1}$ & The total days of $y_{i}<\bar{y}$ \\
\hline$j_{2}$ & The total days of $y_{i}>\bar{y}$ \\
\hline
\end{tabular}

TABLe 3: Data for user 1.

\begin{tabular}{|c|c|c|c|c|c|c|c|c|c|c|c|}
\hline$I_{1}$ & $I_{2}$ & & Day 1 & Day 2 & Day 3 & Day 4 & Day 5 & Day 6 & Day 7 & Day 8 & Day 9 \\
\hline \multirow{3}{*}{$o_{1}$} & $o_{11}$ & & 286 & 290 & 292 & 273 & 285 & 291 & 286 & 280 & 290 \\
\hline & $o_{12}$ & & 208 & 209 & 209 & 211 & 211 & 211 & 211 & 212 & 212 \\
\hline & $o_{13}$ & & 0 & 0 & 0 & 0 & 0 & 0 & 0 & 0 & 0 \\
\hline \multirow{6}{*}{$o_{2}$} & \multirow{2}{*}{$o_{21}$} & $a^{L}$ & 0.24 & 0.36 & 0.36 & 0.34 & 0.36 & 0.4 & 0.4 & 0.42 & 0.42 \\
\hline & & $a^{U}$ & 0.824 & 0.831 & 0.831 & 0.826 & 0.828 & 0.836 & 0.836 & 0.84 & 0.84 \\
\hline & \multirow{2}{*}{$o_{22}$} & $b^{L}$ & 0.324 & 0.326 & 0.326 & 0.325 & 0.326 & 0.328 & 0.328 & 0.328 & 0.33 \\
\hline & & $b^{U}$ & 0.526 & 0.528 & 0.528 & 0.528 & 0.528 & 0.532 & 0.532 & 0.532 & 0.534 \\
\hline & \multirow{2}{*}{$o_{23}$} & $c^{L}$ & 0.120 & 0.122 & 0.122 & 0.122 & 0.122 & 0.124 & 0.124 & 0.126 & 0.126 \\
\hline & & $c^{U}$ & 0.824 & 0.842 & 0.842 & 0.842 & 0.842 & 0.856 & 0.856 & 0.858 & 0.858 \\
\hline \multirow{5}{*}{$o_{3}$} & \multirow{2}{*}{$o_{31}$} & $x^{L}$ & 0.048 & 0.064 & 0.064 & 0.102 & 0.102 & 0.102 & 0.102 & 0.124 & 0.124 \\
\hline & & $x^{U}$ & 0.856 & 0.882 & 0.882 & 0.934 & 0.934 & 0.934 & 0.934 & 0.956 & 0.956 \\
\hline & \multirow{2}{*}{$o_{32}$} & $y^{L}$ & 0.012 & 0.012 & 0.024 & 0.024 & 0.024 & 0.024 & 0.024 & 0.032 & 0.032 \\
\hline & & $y^{U}$ & 0.438 & 0.438 & 0.442 & 0.442 & 0.442 & 0.442 & 0.442 & 0.448 & 0.448 \\
\hline & $o_{33}$ & & 653 & 654 & 656 & 656 & 656 & 658 & 658 & 658 & 660 \\
\hline
\end{tabular}


microblogs, and the original rate of microblogs are selected as the comprehensive index system of microblog influence. Considering the highly dynamic and unstructured characteristics of social network data, the idea of interval intuitionistic fuzzy is introduced to transform the evaluation of social network users' influence into interval intuitionistic fuzzy multiattribute group decision-making problem, and a model is established. Finally, the model is applied to dynamic evaluation of Sina Weibo users' influence to verify the effectiveness of the model.

Different from the method of user influence based on computer iterative operation, the dynamic evaluation model of microblog user influence based on interval intuitionistic fuzzy entropy has the following two characteristics:

(1) Aiming at the limitation of the existing entropy calculation methods, a new IVIF entropy calculation formula based on cosine function is proposed to improve the effectiveness

(2) The user influence evaluation is transformed into fuzzy multiattribute group decision-making problem, which can not only simplify the calculation but also deal with the fuzziness better. It provides a new idea of user influence evaluation and expands the use of fuzzy multiattribute group decision-making

With the rapid development of network information technology [45], people ushered in the era of big data social network. This has brought great changes to our work and life. More and more people like to publish their activities on the Internet. At the same time, information security issues [20, 46] have also been exposed, bringing a series of serious consequences to the people. The limitation of this model is that the selection of microblog user influence evaluation index is not comprehensive enough and information security factors are not considered. This will be further explored in a future work.

\section{Data Availability}

The data used to support the findings of this study are included within the article.

\section{Conflicts of Interest}

The authors declared that they have no conflicts of interest to this work.

\section{Acknowledgments}

This research was supported by the National Natural Science Foundation of China (No. 71661012).

\section{References}

[1] H. Zheng, W. Guo, and N. Xiong, "A kernel-based compressive sensing approach for mobile data gathering in wireless sensor network systems," IEEE Transactions on Systems,
Man, and Cybernetics: Systems, vol. 48, no. 12, pp. 23152327, 2017.

[2] N. Xiong, W. Han, and A. Vandenberg, "Green cloud computing schemes based on networks: a survey," IET Communications, vol. 6, no. 18, pp. 3294-3300, 2012.

[3] N. Xiong, A. Vasilakos, J. Wu et al., "A self-tuning failure detection scheme for cloud computing service," in 2012 IEEE 26th International Parallel and Distributed Processing Symposium, pp. 668-679, Shanghai, China, 2012.

[4] W. Wu, N. Xiong, and C. Wu, "Improved clustering algorithm based on energy consumption in wireless sensor networks," IET Networks, vol. 6, no. 3, pp. 47-53, 2017.

[5] K. Huang, Q. Zhang, C. Zhou, N. Xiong, and Y. Qin, “An efficient intrusion detection approach for visual sensor networks based on traffic pattern learning," IEEE Transaction on Systems, Man, and Cybernetics: Systems, vol. 47, no. 10, pp. 2704-2713, 2017.

[6] J. Li and Z. Li, "Design on MGCF in interworking between IMs and PSTN domain," Computer Networks, vol. 44, no. 23, pp. 62-64, 2018.

[7] Q. Liu, L. Cui, and H. Chen, "Key technologies and applications of internet of things," Computer Science, vol. 37, no. 6, pp. 1-4, 2010.

[8] Z. Qian and Y. Wang, "IoT technology and application," Acta Electronica Sinica, vol. 40, no. 5, 2012.

[9] W. Li, J. Cao, J. Wu, C. Huang, and R. Buyya, "A collaborative filtering recommendation method based on discrete quantuminspired shuffled frog leaping algorithms in social networks," Future Generation Computer Systems, vol. 88, pp. 262-270, 2018.

[10] E. Katz and P. Lazaxsfeld, Personal Influence: The Part Played by People in the Flow of Mass Communications, The Free Press, Illinois, 1955.

[11] W. Zheng, H. Pan, and C. Sun, "A friendship-based altruistic incentive knowledge diffusion model in social networks," Information Sciences, vol. 491, pp. 138-150, 2019.

[12] X. Rui, F. Meng, Z. Wang, and G. Yuan, "A reversed node ranking approach for influence maximization in social networks," Applied Intelligence, vol. 49, pp. 2684-2698, 2019.

[13] Y. Zeng, C. Sreenan, N. Xiong, L. T. Yang, and J. H. Park, "Connectivity and coverage maintenance in wireless sensor networks," The Journal of Supercomputing, vol. 52, no. 1, pp. 23-46, 2010.

[14] W. Guo, N. Xiong, A. V. Vasilakos, G. Chen, and H. Cheng, "Multi-source temporal data aggregation in wireless sensor networks," Wireless Personal Communications, vol. 56, no. 3, pp. 359-370, 2011.

[15] R. He, N. Xiong, L. T. Yang, and J. H. Park, "Using multimodal semantic association rules to fuse keywords and visual features automatically for web image retrieval," Information Fusion, vol. 12, no. 3, pp. 223-230, 2011.

[16] L. Shu, Y. Zhang, Z. Yu, L. T. Yang, M. Hauswirth, and N. Xiong, "Context-aware cross-layer optimized video streaming in wireless multimedia sensor networks," The Journal of Supercomputing, vol. 54, no. 1, pp. 94-121, 2010.

[17] C. Lin, Y. He, and N. Xiong, "An energy-efficient dynamic power management in wireless sensor networks," in 2006 Fifth International Symposium on Parallel and Distributed Computing, pp. 148-154, Timisoara, Romania, 2006.

[18] Y. Zhou, D. Zhang, and N. Xiong, "Post-cloud computing paradigms: a survey and comparison," Tsinghua Science and Technology, vol. 22, no. 6, pp. 714-732, 2017. 
[19] Y. Yang, N. Xiong, N. Y. Chong, and D. Xavier, "A decentralized and adaptive flocking algorithm for autonomous mobile robots," in 2008 The 3rd International Conference on Grid and Pervasive Computing - Workshops, pp. 262-268, Kunming, China, 2008.

[20] A. Shahzad, M. Lee, Y.-K. Lee et al., "Real time modbus transmissions and cryptography security designs and enhancements of protocol sensitive information," Symmetry, vol. 7, no. 3, pp. 1176-1210, 2015.

[21] K. Li, L. Zhang, and H. Huang, "Social influence analysis: models, methods, and evaluation," Engineering, vol. 4, no. 1, pp. 40-46, 2018.

[22] W. Liu, L. Chen, X. Chen, and B. Chen, "An algorithm for influence maximization in competitive social networks with unwanted users," Applied Intelligence, vol. 50, pp. 417-437, 2019.

[23] W. Liu, Y. Li, X. Chen, and J. He, "Maximum likelihood-based influence maximization in social networks," Applied Intelligence, vol. 50, no. 10, pp. 3487-3502, 2020.

[24] X. Chen, C. Zhao, and L. Yang, "A group decision-making model based on interval-valued intuitionistic fuzzy numbers and its application on social network," Systems EngineeringTheorye Practice, vol. 37, no. 7, pp. 1842-1852, 2017.

[25] J. Kleinberg, "Authoritative sources in a hyperlinked environment," Journal of the ACM, vol. 46, no. 5, pp. 604-632, 1999.

[26] X. Kong, F. Xia, Z. Ning et al., "Mobility dataset generation for vehicular social networks based on floating car data," IEEE Transactions on Vehicular Technology, vol. 67, no. 5, pp. 3874-3886, 2018.

[27] K. Atanassov and G. Gargov, "Interval-valued intuitionistic fuzzy sets," Fuzzy Sets and Systems, vol. 31, no. 3, pp. 343349, 1989.

[28] M. Gao, T. Sun, and J. Zhu, "Interval-valued intuitionistic fuzzy multiple attribute decision-making method based on revised fuzzy entropy and new scoring function," Control and Decision, vol. 31, no. 10, pp. 1757-1764, 2016.

[29] $\mathrm{Z}$. Xu, "Methods for aggregating interval-valued intuitionistic fuzzy information and their application to decision making," Control and Decision, vol. 22, no. 2, pp. 215-219, 2007.

[30] Y. Zhao and J. Mao, "New type of interval-valued intuitionistic fuzzy entropy and its application," Computer Engineering and Applications, vol. 52, no. 12, pp. 85-89, 2016.

[31] M. Cha, H. Haddadi, F. Benevenuto, and K. P. Gummadi, "Measuring user influence in twitter: the million follower fallacy," in 4th International AAAI Conference on Weblogs and Social Media, pp. 10-17, Washington DC, USA, 2010.

[32] H. Kwak, C. Lee, H. Park, and S. Moon, "What is twitter, a social network or a news media?," in Proceedings of the 19th international conference on World wide web - WWW '10, pp. 591-600, Raleigh, USA, 2010.

[33] C. Lee, H. Kwak, H. Park, and S. Moon, "Finding influentials based on the temporal order of information adoption in twitter," in Proceedings of the 19th international conference on World wide web - WWW'10, pp. 1137-1138, Raleigh, USA, 2010.

[34] D. Romero, W. Galuba, S. Asur, and B. A. Huberman, "Influence and Passivity in Social Media," in Machine Learning and Knowledge Discovery in Databases. ECML PKDD 2011, D. Gunopulos, T. Hofmann, D. Malerba, and M. Vazirgiannis, Eds., vol. 6913 of Lecture Notes in Computer Science, pp. 1833, Springer, Berlin, Heidelberg, 2011.
[35] J. Weng, E.-P. Lim, J. Jiang, and Q. He, “Twitterrank: finding topic-sensitive influential twitterers," in Proceedings of the third ACM international conference on Web search and data mining - WSDM '10, pp. 261-270, New York, USA, 2010.

[36] S. Ye and F. Wu, "Measuring message propagation and social influence on Twitter.com," International Journal of Communication Networks and Distributed Systems, vol. 11, no. 1, pp. 59-76, 2013.

[37] E. Bakshy, J. Hofman, W. A. Mason, and D. J. Watts, "Everyone's an influencer: quantifying influence on twitter," in Proceedings of the fourth ACM international conference on Web search and data mining - WSDM '11, pp. 65-74, Hong Kong, China, 2011.

[38] K. Atanassov, "Intuitionistic fuzzy sets," Fuzzy Sets and Systems, vol. 20, no. 1, pp. 87-96, 1986.

[39] I. K. Vlachos and G. Sergiadis, "Subsethood, entropy, and cardinality for interval-valued fuzzy sets-an algebraic derivation," Fuzzy Sets and Systems, vol. 158, no. 12, pp. 1384-1396, 2007.

[40] Q. Zhang, S. Jiang, B. Jia, and S. Luo, "Some information measures for interval-valued intuitionistic fuzzy sets," Information Sciences, vol. 180, no. 24, pp. 5130-5145, 2010.

[41] H. Ren and Z. Tan, "Multi-attribute decision making model coupled with interval valued intuitionistic fuzzy entropy-set pair analysis-technique for order preference by similarity to an ideal solution," Control Theory \& Applications, vol. 37, no. 1, pp. 176-186, 2020.

[42] M. Hu, C. Fan, and K. Shi, "Character analysis of standardization methods of decision matrix with intervals," Computer Science, vol. 40, no. 10, pp. 203-207, 2013.

[43] Z. Yue, "An approach to aggregating interval numbers into interval-valued intuitionistic fuzzy information for group decision making," Expert Systems with Applications, vol. 38, no. 5, pp. 6333-6338, 2011.

[44] Y. Yu, J. Yuan, Z. Zhu, and T. Liu, "Evaluation and comparative study on the influence of university official microblog based on PCA and Ca," China Business \& Trade, vol. 25, pp. 167-172, 2018.

[45] W. Guo, N. Xiong, H.-C. Chao, S. Hussain, and G. Chen, "Design and analysis of self-adapted task scheduling strategies in wireless sensor networks," Sensors, vol. 11, no. 7, pp. 6533$6554,2011$.

[46] Q. Zhang, C. Zhou, N. Xiong, Y. Qin, X. Li, and S. Huang, "Multimodel-based incident prediction and risk assessment in dynamic cybersecurity protection for industrial control systems," IEEE Transactions on Systems, Man, and Cybernetics: Systems, vol. 46, no. 10, pp. 1429-1444, 2015. 